\title{
Antibiotic Resistance Pattern in Pseudomonas Aeruginosa Isolated from Different Clinical Specimens
}

\author{
Hoque $\mathrm{MM}^{1}$, Ahmad $\mathrm{M}^{2}$, Khisa $\mathrm{S}^{3}$, Uddin $\mathrm{MN}^{4}$, Jesmine $\mathrm{R}^{5}$
}

\begin{abstract}
Introduction: Pseudomonas aeruginosa is a major clinical microbiological problem affecting the hospitalized and non-hospitalized patients throughout the world. The susceptibility patterns of $P$. aeruginosa vary geographically and with clinical presentation. Pseudomonas can rapidly develop resistance especially when single drug is employed due to frequent mutation and its own innate mechanisms of antibiotic resistance.
\end{abstract}

Objectives: This cross sectional study was carried out to determine in-vitro resistance pattern of Pseudomonas isolates to common antimicrobial agents by disc diffusion method. Various clinical samples were collected from Combined Military Hospital (CMH), Dhaka.

Materials and Methods: This study was carried out in the Department of Microbiology, Armed Forces Institute of Pathology (AFIP) from 01 March 2012 to 31 August 2012. Identification and antibiogram were performed for Pseudomonas isolates following standard microbiological laboratory procedure.

Results: A total of 198 P. aeruginosa were isolated from the various specimens. The highest number (76) of $P$. aeruginosa were isolated from wound swab/pus $(38.38 \%)$, followed by urine $(56,28.28 \%)$, bronchial wash $(23,11.62 \%)$. In present study, maximum number of $P$. aeruginosa are resistant to penicillin $(98.98 \%)$ followed by cephalosporins (89.85\%), aminoglycosides (80.04\%), carbapenems $(76.08 \%)$. The most sensitive antibiotic was combination of piperacillin and tazobactam (only $3.37 \%$ resistant) followed by ciprofloxacin $(54.04 \%)$ and azithromycin (59.18\%).

Conclusion: To prevent the spread of the resistant bacteria, it is critically important to have strict antibiotic policies. The surveillance programmes for multidrug resistant organisms and infection control procedures need to be implemented properly. The antibiotic susceptibility pattern of $P$. aeruginosa needs to be continuously monitored in specialized clinical units and the results readily made available to the clinicians so as to minimize the resistance.

Key-words: Pseudomonas aeruginosa, antibiotic resistance.

\section{Introduction}

Pseudomonas aeruginosa are Gram negative rods that resemble the members of Enterobacteriaceae but differ in that they are strict aerobe and oxidase positive. They are able to grow in water containing only trace of nutrients e.g. tap water and this favors their persistence in the hospital environment. The organisms have been found in hexachlorophene containing soap solutions, in antiseptics and in detergents.

$P$. aeruginosa is found chiefly in soil and water, although approximately $10 \%$ of people carry in the normal flora of the colon. It is found on the skin in the moist area and can colonize the upper respiratory tract of hospitalized patients. Its ability to grow in simple aqueous solutions has resulted in contamination of respiratory therapy and anaesthesia equipment, intravenous fluid, and even distilled water.

$P$. aeruginosa is primarily an opportunistic pathogen. Pathogenesis is based on multiple virulence factors: endotoxin, exotoxin and enzymes. Its endotoxin, like that of other Gram negative bacteria, causes symptoms of sepsis and septic shock. Some strains having "type III secretion system" that transfers the the exotoxin from the bacterium directly into the adjacent human cell which allows the toxin to avoid neutralizing antibody. Some strains causing cystic

1. Lt Col Md Monirul Hoque, MBBS, MCPS, DCP, FCPS, Classified Specialist in Pathology, CMH, Bogra; 2. Lt Col Mushtaq Ahmad, MBBS, DFM, MCPS, FRSPH, Associate Professor \& Head, Department of Forensic Medicine and Toxicology, AFMC, Dhaka; 3. Maj Suman Khisa, MBBS, MCPS, DCP, Graded Specialist in Pathology, AFMI, Dhaka; 4. Col Md Nizam Uddin, MBBS, MCPS, DCP, FCPS, Associate Professor of Pathology, AFMC, Dhaka; 5. Lt Col Rezina Jesmine, MBBS, MCPS, DCP, Graded Specialist in Pathology, AFMI, Dhaka. 
fibrosis have a prominent slime layer (glycocalyx) that mediate adherence to mucous membrane ${ }^{1} . P$. aeruginosa is intrinsically resistant to several antibiotics because of the low permeability of its outer membrane, the constitutive expression of various efflux pumps and the production of antibiotic-inactivating enzymes (e.g. cephalosporinases). Intrinsic resistance involves the collaboration of restricted uptake through the outer membrane and secondary resistance mechanisms such as energy-dependent efflux and $\beta$-lactamases ${ }^{2}$.

\section{Materials and Methods}

This cross sectional laboratory based retrospective study was carried out in the Department of Microbiology, AFIP from 1st March to 31st August 2012. A total of 198 Pseudomonas aeruginosa were isolated from different clinical specimens including wound swab/ pus, urine, bronchial wash, sputum, tracheal aspiration, throat swab, umbilical swab, catheter tip, ear swab, high vaginal swab, pleural fluid, bile etc. The isolates were identified by colony morphology, Gram's staining and biochemical test according to standard laboratory test methods ${ }^{3}$. The antibiotic susceptibility patterns of Pseudomonas isolates were analyzed by clinically used common antibiotics. Susceptibility test was carried out by disc diffusion method (Kirby-Bauer) in Muller-Hinton agar media according to the National Committee for Clinical Laboratory Standards (NCCLS) guidelines ${ }^{4}$. Staph aureus ATCC 25923 was used as control strain. The results of susceptibility test were into susceptible and resistant. The isolates with intermediate susceptibility were included in resistant category.

\section{Results}

In this study, among the 198 isolates of $P$. aeruginosa, 76 were isolated from wound swab/ pus (38.38\%), 56 $(28.28 \%)$ from urine, $23(11.62 \%)$ from bronchial wash, $19(9.59 \%)$ from sputum. The rests are from tracheal aspiration, throat swab, umbilical swab, catether tip, ear swab, HVS, pleural fluid and bile (Table-I).

Table-I: Distribution of the growth of $P$. aeruginosa in different types of clinical specimens $(n=198)$.

\begin{tabular}{|l|c|c|}
\hline Name of specimen & Number of specimens & Percentage (\%) \\
\hline Wound swab/pus & 76 & 38.38 \\
\hline Urine & 56 & 28.28 \\
\hline Bronchial wash & 23 & 11.62 \\
\hline Sputum & 19 & 9.59 \\
\hline Tracheal aspiration & 7 & 3.54 \\
\hline Throat swab & 6 & 3.03 \\
\hline Umbilical swab & 4 & 2.02 \\
\hline Catether tip & 2 & 1.02 \\
\hline Ear swab & 2 & 1.02 \\
\hline HVS & 1 & 0.50 \\
\hline Pleural fluid & 1 & 0.50 \\
\hline Bile & 1 & 0.50 \\
\hline Total & $\mathbf{1 9 8}$ & $\mathbf{1 0 0}$ \\
\hline
\end{tabular}

The resistance rate of $P$. aeruginosa isolated from different specimens to different antimicrobials varied from $3 \%$ to $100 \%$. Organisms isolated from wound swab/pus samples were more resistant to amoxicillin $(98.69 \%)$, cefixime $(96.83 \%)$, gentamicin $(91.90 \%)$, amikacin $(89.19 \%)$, aztreonam $(86.96 \%)$, ceftriaxone $(86.85 \%)$, ceftazidime $(86.85 \%)$. Hundred percent sensitivity was shown to combined antibiotics piperacillin and tazobactam (Table-II). Among the organisms isolated from urine, the rate of resistance was $100 \%$ for amoxicillin, $98.22 \%$ for azithromycin, $98.12 \%$ for cephradine, $97.78 \%$ for carbenicillin, $89.29 \%$ for ceftriaxone. The most sensitive drug was combination of piperacillin and tazobactam (sensitivity 95\%) (Table-II). In case of isolates from bronchial wash, $100 \%$ resistance rate was found against cefuroxime and cefixime. The most effective drug was combination of pipericillin and tazobactam (87.5\% sensitive) (Table-II). In case of isolates from sputum, $100 \%$ resistance rate was seen against amoxicillin and $100 \%$ sensitivity was seen against pipericillin and tazobactam combination. Ciprofloxacin was found very effective (sensitivity $89.47 \%$ ) (Table-II). 
In isolates of tracheal aspirates, P. aeruginosa showed $100 \%$ resistance against amoxicillin, ciprofloxacin, ceftriaxone, ceftazidime, carbenicillin, cephradine and cefuroxime but opposite happened for combination of pipericillin and tazobactam (Table-II).

Hundred percent resistance rate was found against amoxicillin, cephradine and cefuroxime. But all the isolates of umbilical swab were sensitive to ceftriaxone, ciprofloxacin, ceftazidime, azithromycin, carbenicillin and combined antibiotics ( pipericillin + tazobactam) (Table-II). All P. aeruginosa isolates from throat swab were $100 \%$ resistant to amoxicillin, ceftriaxone and cefuroxime. All were sensitive to ciprofloxacin and combined antibiotic (piperacillin+ tazobactam) (Table-II).

Table-II: Resistance rates of Pseudomonas aeruginosa to different antibiotics ( $n=198)$.

\begin{tabular}{|l|c|c|c|c|c|c|c|c|}
\hline Antibiotic & $\begin{array}{c}\text { Wound swab } \\
\mathbf{n = 7 6 ( \% )}\end{array}$ & $\begin{array}{c}\text { Urine } \\
\mathbf{n}=\mathbf{5 6 ( \% )}\end{array}$ & $\begin{array}{c}\text { Bronchial wash } \\
\mathbf{n}=\mathbf{2 3 ( \% )}\end{array}$ & $\begin{array}{c}\text { Sputumn } \\
\mathbf{n = 1 9 ( \% )}\end{array}$ & $\begin{array}{c}\text { Tracheal aspirate } \\
\mathbf{n = 0 7 ( \% )}\end{array}$ & $\begin{array}{c}\text { Throat swab } \\
\mathbf{n = 0 6 ( \% )}\end{array}$ & $\begin{array}{c}\text { Umbilical swab } \\
\mathbf{n}=\mathbf{0 4}(\%)\end{array}$ & $\begin{array}{c}\text { Total } \\
\mathbf{1 9 8}(\%)\end{array}$ \\
\hline Amoxicillin & $75(98)$ & $56(100)$ & $22(95)$ & $19(100)$ & $7(100)$ & $6(100)$ & $4(100)$ & $196((98)$ \\
\hline Ciprofloxacin & $47(61)$ & $47(83)$ & $1(4)$ & $2(10)$ & $7(100)$ & $0(0)$ & $0(0)$ & $107(54)$ \\
\hline Ceftriaxone & $66(86)$ & $50(89)$ & $16(69)$ & $15(78)$ & $7(100)$ & $6(100)$ & $0(0)$ & $164(82)$ \\
\hline Ceftazidime & $66(86)$ & $52(92)$ & $14(60)$ & $17(89)$ & $7(100)$ & $2(33)$ & $0(0)$ & $162(81)$ \\
\hline Azithromycin & $36(47)$ & $55(98)$ & $8(34)$ & $8(47)$ & $1(14)$ & $4(66)$ & $0(0)$ & $116(59)$ \\
\hline Carbenicillin & $62(81)$ & $44(97)$ & $10(43)$ & $15(78)$ & $7(100)$ & $4(66)$ & $1(25)$ & $147(78)$ \\
\hline Aztreonam & $40(86)$ & $35(87)$ & NT & $3(50)$ & $6(85)$ & NT & NT & $86(84)$ \\
\hline Netilmicin & $25(71)$ & $28(68)$ & NT & NT & $5(71)$ & NT & NT & $60(70)$ \\
\hline Gentamicin & $34(91)$ & $34(82)$ & NT & NT & $6(85)$ & NT & NT & $75(85)$ \\
\hline Amikacin & $33(89)$ & $31(79)$ & NT & NT & $5(71)$ & NT & NT & $70(81)$ \\
\hline Meropenem & $27(69)$ & $32(80)$ & $7(77)$ & $2(20)$ & $6(85)$ & $1(25)$ & NT & $76(67)$ \\
\hline Cephradine & NT & $53(98)$ & $16(94)$ & $16(84)$ & $7(100)$ & $5(83)$ & $4(100)$ & $104(94)$ \\
\hline Cefixime & $61(96)$ & $25(71)$ & $23(100)$ & NT & NT & NT & NT & $109(90)$ \\
\hline Cefuroxime & NT & NT & $23(100)$ & NT & $7(100)$ & $6(100)$ & $4(100)$ & $40(100)$ \\
\hline Pipericillin+Tazobactam & $0(0)$ & $1(5)$ & $2(12)$ & $0(0)$ & $0(0)$ & $0(0)$ & $0(0)$ & $3(3)$ \\
\hline
\end{tabular}

${ }^{* *}$ Ear swab (n=2), Catheter tip (n=2), HVS ( $\left.n=1\right)$, Pleural fluid $(n=1)$, Bile $(n=1)$ were not shown in the table. *NT= Not tested.

When all samples considered together, maximum resistance was seen against cefuroxime $(100 \%)$ followed by amoxicillin (98.98\%), cephradine (94.54\%), cefixime (90.08\%), gentamicin $(85.22 \%)$, aztreonam $(84.31 \%)$, ceftriaxone $(82.82 \%)$, ceftazidime $(81.82 \%)$, amikacin $(81.40 \%)$ etc. Most sensitive antibiotic was combination of piperacillin and tazobactam (resistance rate $3.37 \%$ ) (Table-II).

\section{Discussion}

Pseudomonas aeruginosa emerged as an important pathogen and responsible for the nosocomial infections. It is one of the important causes of morbidity among hospital patients. The increasing isolation rate of Pseudomonas aeruginosa in hospital infections is due to its resistance to common antibiotics and antiseptics, and its ability to establish itself widely in hospitals. In present study it is evident that there is distinct difference in the sensitivity pattern of isolates of Pseuodomonas aeruginosa from specimen to specimen. In this study, the maximum clinical isolates of $P$. aeruginosa were isolated from pus/swab $(38.38 \%)$, followed by urine $(28.28 \%)$. These results are in line with studies of Jamshaid AK et al. ( pus $57.64 \%$ and urine $24.2 \%)^{5}$. In this study, isolates from wound swab/pus were more resistant than other isolates. Wound/pus swab isolates were resistant to amoxicillin $98.69 \%$, ceftriaxone $86.85 \%$, ceftazidime $86.85 \%$. Same pattern of resistance was observed in a study by Mohiuddin et al. in Bangladesh ${ }^{6}$. Previously in a study carried out in AFIP the resistance rate of ceftriaxone, ceftazidime and aztreonam were $42.73 \%, 25.45 \%$ and $8.18 \%$ respectively $^{7}$. In urine sample, it has been found amoxicillin was fully resistant and the resistance rate for ceftriaxone and ciprofloxacin were $89.29 \%$ and $83.93 \%$. A study carried out in AFIP, Bangladesh showed same 100\% resistance to amoxicillin but lower resistance to ceftriaxone and ciprofloxacin $(20.83 \% \text { and } 29.16 \%)^{7}$. The increased resistance to all antibiotic is frustrating. The resistance pattern found in bronchial wash, sputum, throat swab and tracheal aspirates are also 
very alarming. Resistance to aminoglycosides were higher in other parts of the world and $70.70 \%$ by Lutfu Savas et al. and $69.86 \%$ by Agandi KM et $a^{8,9}$. In this study, it was also higher $(85.22 \%)$. For amikacin, recorded resistance was $81.40 \%$ where as lower rate were reported from Pakistan $(6.73 \%$ by Nadeem et al. and $24 \%$ by Jamshaid et al. $)^{10,5}$. A study in India showed by Meenakumari, the rate was $56.63 \%{ }^{11}$. The variations may be related to the acquisition of drug resistant genes and patterns of antibiotic prescription for a particular location. In present study, resistances to cephalosporins were observed more than $80 \%$ (cephradine $94.54 \%$, ceftriaxone $82.82 \%$, ceftazidime $81.82 \%$, cefuroxime $100 \%$, cefixime $90.08 \%$ ). Lower rate of resistance was explored in our institute (AFIP) ${ }^{7}$ (ceftriaxone $32.46 \%$, ceftazidime $17.28 \%$ ) in 2004 . Lower rates were also observed by Nicholas et al. (6\%), Paul et al. $(19.6 \%)$, Farida et al. $(38 \%)^{12,13,14}$. Remarkable resistance to third generation cephalosporins were also recorded (>90\%) by Bhale Rao et al. and $(96-100 \%)$ by Awari et al in India ${ }^{15,16}$.

Resistance to piperacillin + tazobactam combination was observed in only $3.37 \%$ of our isolates; higher rates of $45.6 \%$ were observed in Angadi K M et al ${ }^{9}$. It was $9.6 \%$ in 2007 by Nadeem et $\mathrm{al}^{10}$. The lower resistance rate in this study may be due to recent introduction of these combinations as antipseudomonal therapy. Resistance to meropenem is $67.85 \%$ in this study. Same higher resistance rate was also found in two Indian studies ${ }^{11,17}$. In present study, resistance to aztreonam was $84.32 \%$. But $100 \%$ resistance rate was seen by S. Meenakumari et al. in India ${ }^{11}$. The only drug tested in fluoroquinolone group is ciprofloxacin. It has been observed the resistance rate was $54.04 \%$. Same line of resistance was observed in two other Indian studies $^{9,11}$. In present study, resistance rate for azithromycin was $59.18 \%$. But it was found $85.71 \%$ in Gujrat, India ${ }^{17}$. This study revealed that the clinical isolates of Pseudomonas aeruginosa are becoming resistant to commonly used antibiotics and gaining more and more resistance to newer antibiotics. Still pipericillin and tazobactam synergy is the most effective combination for anti-pseudomonal therapy as we observed in our study. The antimicrobial agents are losing their efficacy because of the spread of resistant organisms due to indiscriminate use of antibiotics, lack of awareness, patient's noncompliance and unhygienic condition.

\section{Conclusion}

In fact, the irrational and inappropriate use of antibiotics is responsible for the development of resistance of Pseudomonas species to antibiotic monotherapy. Hence, there is a need to emphasize the rational use of antimicrobials and strictly adhere to the concept of "reserve drugs" to minimize the misuse of available antimicrobials. To prevent the spread of the resistant bacteria, it is critically important to have strict antibiotic policies while surveillance programmes for multidrug resistant organisms and infection control procedures need to be implemented. It is desirable that the antibiotic susceptibility pattern of bacterial pathogens like $P$. aeruginosa in specialized clinical units to be continuously monitored and the results readily made available to clinicians so as to minimize the resistance and morbidity.

\section{References}

1. Levinson W, Jawetz E. Medical Microbiology and Immunology, Examination and Board review 11th ed. New York: McGraw-Hill; 2010: 137-8.

2. Hancock REW. The bacterial outer membrane as a drug barrier. Trends Microbiology. 1997; 5: 37-42.

3. Collee G, Fraser AG, Marimion BP et al. Practical Medical Microbiology. 14th ed. New York: Churchill Livingstone: 1996; 413-24.

4. Natinal Committee for Clinical Laboratory Standards: Performance standards for antimicrobial disc susceptibility tests. Approved standard M2A7. NCCLS. Villanova. PA 1995; 15(14).

5. Jamshaid AK, Zafar I, Saeed UR et al. Prevalence and resistance patterns of Pseudomonas aeruginosa against various antibiotics. Pak J. Pharma. Sci. 2008; 21:311-5.

6. Mohiuddin M, Haq JA, Haq MM et al. Microbiology of Nosocomial Infection in Tertiary Hospitals of Dhaka City and Its Impact. Bangladesh Journal of Medical Microbiology. 2010; 04 (02): 32-8.

7. Wadud A, Rahman M, Wasey AFSA. Antibiotic resistance in Pseudomonas aeruginosa strains isolated from various clinical specimens. Bangladesh Armed Forces Medical Journal. June 2004; 34: 31-5. 
8. Savas L, Duran N, Savas N et al. The prevalence and Resistance Patterns of Pseudomonas aeruginosa in Intensive Care Units in a University Hospital. Turkey Journal of Medical Science. 2005; 35:317-22.

9. Angadi KM, Kadam M, Modakn MS et al. Detection of Antibiotic Resistance in Pseudomonas aeruginosa Isolates With Special Reference to Metallo $\beta$-Lactamases From a Tertiary Care Hospital In Western India. International Journal of Microbiology Research. 2012; 14(7):295-8.

10. Nadeem, Raja S, Singh NN. Antibiotic Susceptibility Pattern of Clinical Isolates of Pseudomonas aeruginosa in a Tertiary Care Hospital. Journal of Microbiology, Immunology and Infection. 2007; 40: 45-9.

11. Meenakumari S, Verma S, Absar A et al. Antimicrobial Susceptibility Pattern of Clinical Isolates of Pseudomonas aeruginosa in an Indian Cardiac Hospital. International Journal of Engineering Science and Technology. September 2011; 3:7117-23.

12. Troillet N, Samore MH, Carmeli Y. Imipenemresistant Pseudomonas aeruginosa: risk factors and antibiotic susceptibility patterns. Clin Infect Dis 1997; 25:1094-8.
13. Brown PD, Izundu A. Antibiotic Resistance in Clinical Isolates of Pseudomonas aeruginosa in Jamaica. Rev Panam Salud Publica/Pan Am J Public Health 2004;16(2):125-30.

14. Anjum F, Munir A. Susceptibility Pattern of Pseudomonas aeruginosa against Various Antibiotics. African Journal of Microbi-ology Research. 2010 : 4(10) 1005-12.

15. Bhalerao D, Roushani S, Kinikar AG. Study of Metallo-beta lactamase producing Pseudomonas aeruginosa in Pravara Rural Hospital.Pravara Medical Review. 2010; 5(3): 16-9.

16. Abhijit $A$ and Nighute $S$. Incidence of Metallobetalactamase Producing Pseudomonas aeruginosa in Kesar SAL Medical College and Hospital, Ahmedabad. International Journal of Biomedical Research. 2012; 23(11): 32-5.

17. Javiya VA, Somsuvra B, Ghatak SB et al. Antibiotic susceptibility patterns of Pseudomonas aeruginosa at a tertiary care hospital in Gujarat, India. Indian Journal of Pharmacology. 2008; 40(5): 230-4. 